\title{
Analyzing 5G RF System Performance and Relation to Link Budget for Directive MIMO
}

\author{
Tommi Tuovinen, Nuutti Tervo, Student Member, IEEE, and Aarno Pärssinen, Senior Member, IEEE
}

\begin{abstract}
Wideband fifth generation (5G) systems utilizing high carrier frequency and multiple-input multiple-output (MIMO) raise major challenges for the system design. Wave propagation and practical hardware tradeoffs at higher frequency ranges provide new boundary conditions for the implementation. This paper addresses system performance boundaries and the analysis method towards multibeam communications at mmW. We combine analysis from antennas and propagation to the RF transceiver specifications and beamforming requirements. Realistic propagation model and antenna implementation is used to generate beam-specific path gains and provide a wide variety of user scenarios. Using this approach, system level interdependencies and RF performance boundaries can be verified with different antenna configurations in various propagation environments. As an example, we present MIMO link budget analysis targeting $10 \mathrm{Gbits} / \mathrm{s}$ for multiple devices in the office scenario at $27 \mathrm{GHz}$.
\end{abstract}

Index Terms-Antenna system, behavioral modelling, propagation, system analysis, performance analysis, RF, transceiver.

\section{INTRODUCTION}

$\mathrm{E}$ XTENSIVE utilization of multiple-input multiple-output (MIMO) [1],[2] is one of the fundamentals in the operation of the fifth generation (5G) communications. Recent years have shown a significant growth of user's need for data rate in cellular communications and the demands seem to only escalate [3]. Several companies have shared the outlook of peak data rates lying in the range of ten Gbits/s with the targeted cell edge rates not lower than a Gbit/s [4],[5]. To achieve the rate requirements, system bandwidth needs to be widened and several spatially multiplexed data streams (SMDSs) are required even for individual users. In addition to the selections in World Radio Communication conference (WRC)-15 [6], academic research and industrial companies have indicated growing consensus that millimeter wave $(\mathrm{mmW})$ region will play a central role in 5G [7]-[9]. Increasing the center frequency decreases the antenna aperture, which is the reason why the antenna directivity needs to be utilized, e.g., to achieve reasonable link range.

This research work was supported by Finnish Funding Agency for Technology and Innovation (TEKES), Nokia Networks, Huawei Technologies and Keysight Technologies. The work of N. Tervo was supported by Infotech Oulu Graduate School.

The authors are with Centre for Wireless Communications (CWC) Radio Technologies, University of Oulu, Oulu, FI-90014 Finland (e-mail: tommi.tuovinen@oulu.fi; nuutti.tervo@oulu.fi; aarno.parssinen@oulu.fi).
Utilizing MIMO at $\mathrm{mmW}$ will inevitably set major constraints for a realistic 5G radio frequency (RF) implementation in terms of form factors and system bandwidth. The beam-centric propagation environment is reforming the antenna design and one needs to be adaptive for providing gain to several directions simultaneously. Although a base station (BS) can be designed for a specific scenario, a mobile terminal (MT) needs to be adaptive for various propagation environments. In the open literature, arrays have been most popular approach to provide antenna gain for $5 \mathrm{G}$ configurations [10]-[12]. However, it is evident that arrays for MTs are strongly limited because of mechanical restrictions. In addition, transceivers must be integrated close to antennas to avoid notable wiring losses and to achieve a compact form factor. Furthermore, the production of high output power from solidstate circuits with decent linearity, efficiency, and area is complicated in mmWs. Practical solutions are leading to highly integrated modules where RF transceiver with electrical beam control is implemented using RF integrated circuit (RFIC) and connected to antenna using minimum possible wiring to avoid losses [13],[14].

The antenna size will be reduced along the wavelength and the size of an element in arrays will be first comparable and eventually smaller than RF electronics when approaching to mmWs. For instance, the size of a power amplifier (PA) should match to the antenna dimensions in a reasonable manner, which has a direct impact on the maximum power delivery of one element. Since $5 \mathrm{G}$ will require significantly more DSP power due to very high data rates, careful antenna, RF and DSP cooptimization is required for power efficient solutions. In the RF system design and optimization, physically inconsistent assumptions would be observed as smaller user-experienced data rates and ranges than envisioned. From that perspective, antennas and propagation are essential factors on providing realistic RF system requirements. Cellular radio requirements for mmW MIMO systems are still open although discussions on the fundamental factors are on-going in several forums including the $3^{\text {rd }}$ Generation Partnership Project (3GPP) [15]. The focus of this paper is to present methods for defining RF and antenna requirements and performance boundaries together with realistic propagation environment. Key contributions are: (1) abtracted means to combine signal processing requirements, RF transceiver performance boundaries, antennas and radio channel to the same model, and (2) estimate and categorize different propagation paths (as independent beams or MIMO 


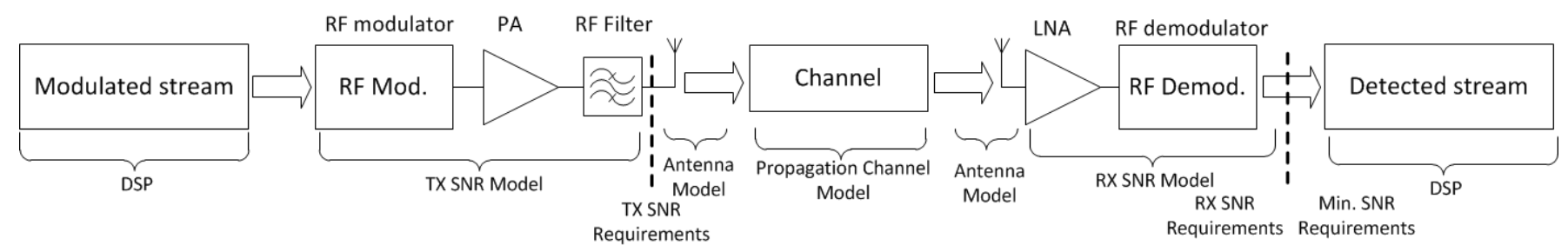

Fig. 1. Communication system model.

channels) for defining link budget in multi-beam scenario.

The paper is structured as follows. In Chapter II, we describe the system model and system level targets for the link-budget. Chapter III describes the transceiver models. In Chapter IV, we describe used antenna model and channel model. Mapping of signal-to-noise ratio (SNR) requirements to RF implementation and multi-stream link budget is presented in Chapter V. Chapters VI and VII presents the discussion and conclusion.

\section{COMMUNICATION SYSTEM MODEL DESCRIPTION}

\section{A. Overview of Link-level Model}

For mmW MIMO system requirement analysis, each part of the communication system must be included using appropriate abstraction in system model for achiving realistic performance results. An overview of the required blocks are given in Fig. 1. Waveform parameters given in Section II-B are needed for estimating digital system performance and RF waveform. RF implementation models represent the impacts of typical RF nonidealities (such as phase noise, I/Q (in-phase / quadrature phase ) imbalance, asymmetry between in a reciprocal link etc.). The TX model includes error vector magnitude (EVM) specifications for a digital front-end (DFE), RF modulator, power amplifier (PA) and RF filter. The RX model consist of noise figure (NF) and peak SNR limitations dominated by nonnoise RF nonidealities. Details of the transmitter (TX) and receiver (RX) models are explained in Chapter III. Throughout the paper, the relation between EVM (as a factor) and SNR (dB) can be simply written as

$$
\mathrm{SNR}=20 \log _{10}\left(\frac{1}{\mathrm{EVM}}\right)
$$

Wave propagation and array models presented in Chapter $\mathrm{V}$ are needed to indicate available path directions to utilize beamforming (BF), and dictate beam-specific path gains for each SMDS.

\section{B. Waveform and Modulation Scheme}

We consider time-division duplex (TDD) system with orthogonal frequency-division multiplexing (OFDM) waveform. System level parameters and assumptions are collected in Table I. The achievable data rate, $R_{\mathrm{Max}}$, which can be scaled to system bandwidth and modulation scheme as [16]

$$
R_{\mathrm{Max}}=\frac{r\left(N_{\mathrm{FFT}}-N_{\mathrm{ACI}}\right) \log _{2}\left(M_{\mathrm{Mod}}\right)}{t_{\mathrm{OFDM}}}
$$

where $r$ denotes coding rate, $N_{\mathrm{FFT}}$ is the size of Fast Fourier Transform (FFT), $N_{\mathrm{ACI}}$ is the number of guard band subcarriers for mitigating the adjacent channel interference (ACI), $M_{\text {Mod }}$ is the modulation order and $t_{\text {OFDM }}$ is the symbol duration including
TABLE I

COMMUNICATION SYSTEM MODEL PARAMETERS AND ASSUMPTIONS

\begin{tabular}{ll}
\hline \hline System & TDD OFDM \\
Waveform & OFDM \\
Link adaptation & BPSK, QPSK, 16QAM, \\
System bandwidth: $B$ & $1000 \mathrm{MHz}$ at 27 GHz \\
FFT size: $N_{\mathrm{FFT}}$ & 8192 \\
Occupied sub-carriers & 7500 \\
Guard band subcarriers: $: N_{\mathrm{ACI}}$ & 692 \\
Sub-carrier spacing & $120 \mathrm{kHz}$ \\
OFDM symbol duration: $t_{\mathrm{OFDM}}$ & $8.83 \mu \mathrm{s}$ \\
Modulation order: $M_{\mathrm{Mod}}$ & $2,4,16,64,256$ \\
Protocol efficiency & $90 \%$ \\
Bandwidth efficiency & $90 \%$ \\
Coding rate: $r$ & $1 / 3,1 / 2,2 / 3,3 / 4,5 / 6,7 / 8,1$ \\
Channel state information & Available in each radio in the \\
Mobility & communication system \\
\hline
\end{tabular}

TABLE II

ACHIEVABLE DATA RATE w/O IMPACT OF CODING

\begin{tabular}{cccccc}
\hline \hline \multirow{2}{*}{$\begin{array}{c}\text { Number of } \\
\text { SMDSs }\end{array}$} & \multicolumn{5}{c}{ Maximum theoretical data rate [Gbit/s] } \\
\cline { 2 - 6 } & BPSK & QPSK & 16QAM & 64QAM & 256QAM \\
\hline 1 & 0.76 & 1.53 & 3.06 & 4.59 & 6.11 \\
2 & 1.53 & 3.06 & 6.11 & 9.17 & 12.23 \\
3 & 2.28 & 4.59 & 9.18 & 13.78 & 18.33 \\
4 & 3.04 & 6.12 & 12.24 & 18.36 & 24.44 \\
\hline
\end{tabular}

TABLE III

MINIMUM SNR REQUIREMENTS FOR GIVEN MODULATION SCHEME

\begin{tabular}{cccccc}
\hline \hline \multirow{2}{*}{$\begin{array}{c}\text { coding } \\
\text { rate } \mathrm{r}\end{array}$} & BPSK & QPSK & 16QAM & 64QAM & 256QAM \\
\cline { 2 - 6 } & 2.2 & 5.2 & 12.7 & 19.2 & 25.2 \\
$1 / 3$ & 4.0 & 7.0 & 14.5 & 21.0 & 27.0 \\
$1 / 2$ & 5.2 & 8.2 & 15.7 & 22.2 & 28.3 \\
$2 / 3$ & 5.8 & 8.8 & 16.3 & 22.8 & 28.8 \\
$3 / 4$ & 6.4 & 9.2 & 16.7 & 23.2 & 29.2 \\
$5 / 6$ & 6.4 & 9.4 & 16.9 & 23.4 & 29.4 \\
$7 / 8$ & 7.0 & 10.0 & 17.5 & 24.0 & 30.0 \\
1 & & & & & \\
\hline
\end{tabular}

cyclic prefix. Bandwidth efficiency for the data rate is equivalent to the long term evolution (LTE) specifications. Maximum theoretical data rates for different modulation schemes without coding is given in Table II. The maximum rate for single stream requires 256QAM which is extremely challenging to achieve with realistic receiver RX and TX nonidealities in practice. On the contrary, more than $10 \mathrm{Gbit} / \mathrm{s}$ can be achieved with lower order modulations.

\section{SNR requirements for Extreme Data Rates}

When increasing the modulation order, the required SNR also increases. Moreover, closer the symbols are to each other in the constellation, the higher SNR is required for achieving decent bit error rate in the RX. Increasing the coding rate decreases the SNR requirement, but it also decreases the maximum data rate. 
Hence, coding enables optimization of the data rate for a certain SNR, like Shannon-Hartley theorem predicts. The required SNR for given modulation is usually formed by setting requirement for symbol error rate (SER) to be around $10^{-3}$. By using the well-known Q-function, the minimum SNR requirement, $S N R_{\text {min }}$, for each modulation and coding rate in the reception can be observed from Table III. When specifying the transceiver, this SNR should be a combined result from both RX and TX, including all RF nonidealities, antennas and radio channel, explained in the following chapters.

\section{RF TRANSCEIVER MODELS AND LINK ADAPTATION}

$\mathrm{RF}$ transceiver performance is limited by a set of different parameters and dominant factors, which vary along the operation range as a function of power. The power dependence of SNR or EVM must be considered in sufficient detail once building the abstraction or tuning model for specific implementation or boundary conditions in mind. Model should be more complex than white noise only in the RX and fixed TX EVM. Such models are valid only at very limited input/output power regimes or link ranges. The models for TX and RX include also non-linear behavior, especially if SNR is calculated as a combination of different nonidealities at each power level at a time.

Used models contain nonidealities (noise and distortion) that have different dependence on the actual signal: (i) signal independent, i.e., absolute power (like white noise), (ii) linearly signal dependent (like I/Q imbalance) and (iii) non-linearly signal dependent (like distortion in power amplifiers). Abstracted modelling can be based on the fact that relatively small errors will be done in most cases if all of these nonidealities are summed together as root-mean-square (RMS) powers rather than using fully accurate amplitude and phase response of each element. In a practical design, nonidealities very rarely sum coherently and there are input/output power regions where only one or two of these components are dominant at a time.

\section{A. TX SNR Model}

The TX model is composed of three abstracted elements as given in Table IV. The digital part including the digital-toanalog converter (DAC) is modelled with the constant EVM (signal independent) as signals are scaled to the optimal level at the DAC output. For downlink (DL), 1\% digital EVM is budgeted. That corresponds to SNR of $40 \mathrm{~dB}$ resulting around 8 to 9 bits minimum resolution for DAC which has two extra bits reserved for the crest factor of the signal to avoid additional clipping in the interface. Digital resolution (i.e. word length) in DSP can be significantly better and therefore not fundamentally limiting the performance. The RF modulator consists of all analog/RF functionality of the TX except actual PA, RF filtering, and switches (RF front-end, RFFE) required before the antenna. Based on a recent example in [19], even an EVM of $0.5 \%$ would be possible for a BS TX. Correspondingly, somewhat lower EVM of $\sim 1 \%$ has been reported for an IC targeted for mobile applications [20]. These numbers represent measured combination of all nonidealities coming from IC so-
TABLE IV

TRANSMITTER EVM IN DL \& UL IN COMMUNICATION MODEL

\begin{tabular}{cccc}
\hline \hline & \multicolumn{3}{c}{ Transmitter blocks } \\
\cline { 2 - 4 } & $\begin{array}{c}\text { Digital } \\
\text { (incl. DAC) }\end{array}$ & $\begin{array}{c}\text { RF modulator } \\
\text { (RFIC) }\end{array}$ & $\begin{array}{c}\text { PA + RF filter } \\
\text { (RFFE) } *^{*}\end{array}$ \\
\hline $\begin{array}{c}\text { DL (BS): EVM @ } \\
\text { [30 dBm] } P_{\text {out }}[\%]\end{array}$ & 1.0 & 0.75 & 2.0 \\
Casc. EVM [\%] & 1.0 & 1.25 & 2.36 \\
UL (UE): EVM @ & 1.5 & 1.0 & 5.5 \\
[2 dBm] $P_{\text {out }}[\%]$ & 1.5 & 1.8 & 5.8 \\
Casc. EVM [\%] & & & \\
\hline
\end{tabular}

*) Maximum requirement for the highest modulation

lution, and are typically dominated by phase noise of the local oscillator and I/Q imbalance (caused by internal amplitude and phase errors) even if calibrated. Both are linearly dependent on the output power resulting to constant EVM over the power range. It is expected that this performance could be achieved also at mmW regime although that will be very challenging. If that appear not to be viable that will result in lower data rates due to the additional coding required.

In practice, TX SNR model is also nonlinear and with proper transceiver design, non-linearity is dominated strongly by the PA behavior. In Table IV, the PA behavior is presented for fixed output power level of $30 \mathrm{dBm}$. The EVM degradation to large signal non-linearity requires a PA device specific model as a function of power. The commercial mmW PA [21] data was extracted from the data-sheet and simulated in AWR simulator using a 256-QAM modulated OFDM signal. RFFE EVM contains also some contribution from non-linear behavior of RF switches and filters although they have typically much better linearity than the PA itself. The RF filter and other losses of $3 \mathrm{~dB}$ are subtracted from output power and embedded to each PA in DL to correspond the implementation aspects between PA and antenna. The used model for RFFE as a function of average output power is shown in Fig. 2 with red line. In UL, we scaled the same output power dependent behavior to the 28$\mathrm{GHz}$ PA in [22], which is a good approximation of a potential solution for 5G MT with state-of-the-art performance. Signal loss of $4 \mathrm{~dB}$ between MT PA and antenna is assumed.

Finally, digital, RF modulator, and RFFE performances can be calculated together simply by

$$
\mathrm{EVM}_{\mathrm{TOT}}=\sqrt{\left(\mathrm{EVM}_{\mathrm{DIG}}^{2}+\mathrm{EVM}_{\mathrm{RFM}}^{2}+\mathrm{EVM}_{\mathrm{RFFE}}^{2}\left(\mathrm{P}_{\mathrm{TX}}\right)\right)}
$$

where $E_{\text {DIG }}, \mathrm{EVM}_{\mathrm{RFM}}$ and $\mathrm{EVM}_{\mathrm{RFFE}}$ present EVM's of digital, RF modulator and RFFE (incl. PA), respectively. The combined models for DL presented as SNRs (dB) according to (1) are presented in Fig. 2. As PA EVM is the only term that has output power dependency, it dominates at high power levels while other EVM terms set floor for the best possible EVM of the TX.

\section{B. RX SNR Models}

In the RX model, the SNR dependence on signal power must be modelled differently than in TX. At low signal levels, noise is the dominant component and the SNR increases linearly as a function of input power. At high power levels, signal dependent nonidealities such as phase noise and I/Q imbalance sets an up 


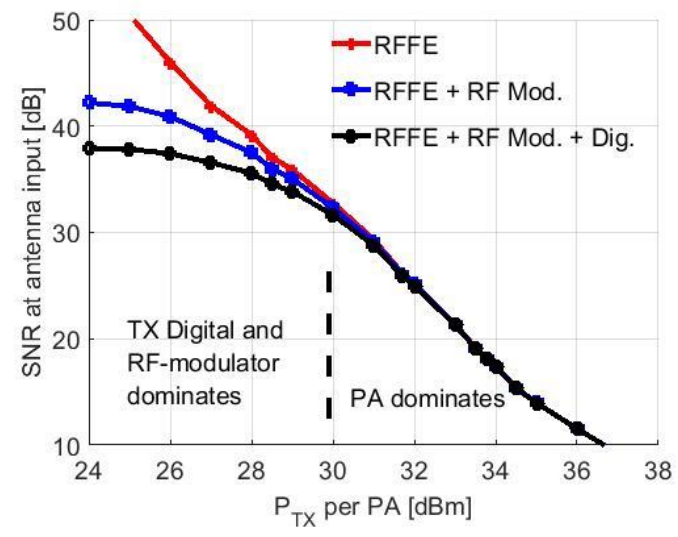

Fig. 2. BS TX EVM (SNR) model as a function of PA average output power.

per boundary for SNR. The latter is dominant constraint for high-order modulations and must be included to models targeting for the peak data rates. Finally, also RX behavior gets non-linear as a function of input power due to the compression. However, RX nonlinearity is ignored here because RX can be made linear enough for the whole signal range using automatic gain control (AGC). Noise dependent terms for RX SNR can be written as

$$
\left.S N R_{\mathrm{n}}(\mathrm{dB})=P_{R X}(\mathrm{dBm})\right]-\left(10 \log _{10}\left(k T_{0} B\right)+N F\right)
$$

where $P_{\mathrm{RX}}$ is the received power in $\mathrm{dBm}, k$ is the Boltzmann's constant, $T_{0}$ is the nominal temperature (typically $290 \mathrm{~K}$ ), $B$ is the system bandwidth and $N F$ is the cascaded NF of the whole receiver in $\mathrm{dB}$.

At the RX, signal dependent nonidealities are basically similar as in TX but somewhat lower performance is expected because calibration in the RX is a more complex procedure to execute. Parameters for the RX are presented in Table V. Although the model contains several parameters related to ADC requirements, with the appropriate gain of the $\mathrm{RX}$, including signal level dependent gain control, the ADC won't have significant impact to the total SNR. In modern wireless systems, this is the common way to design a RX partition. Thus, we can write SNR for RX referred to antenna input as

$$
S N R_{R X}=\frac{1}{\frac{1}{S N R_{n}}+\frac{1}{S N R_{\operatorname{Max}}}}
$$

where $\mathrm{SNR}_{\mathrm{Max}}$ describes the signal dependent nonidealities and $\mathrm{SNR}_{\mathrm{n}}$ is coming from the white noise according to (4). Effects of quantization noise, thermal noise and other nonidealities are presented in Fig. 3. Minor contributions of the ADC quantization noise are shown as dashed line.

The small difference between DL and UL behavior in NF has been included to the model as $N F_{\mathrm{RX}, \mathrm{DL}}=10 \mathrm{~dB}$ and $N F_{\mathrm{RX}, \mathrm{UL}}=$ $8 \mathrm{~dB}$. These numbers include both the internal losses between antenna and RX as well as the thermal noise from the RX itself. As discussed before, the ADC and DSP needs to be specified such that the impact to SNR is negligible. Model described here is only for the in-band operation. More complex models can be developed in similar fashion for out-of-channel interferers when work is extended to RF interference modelling.

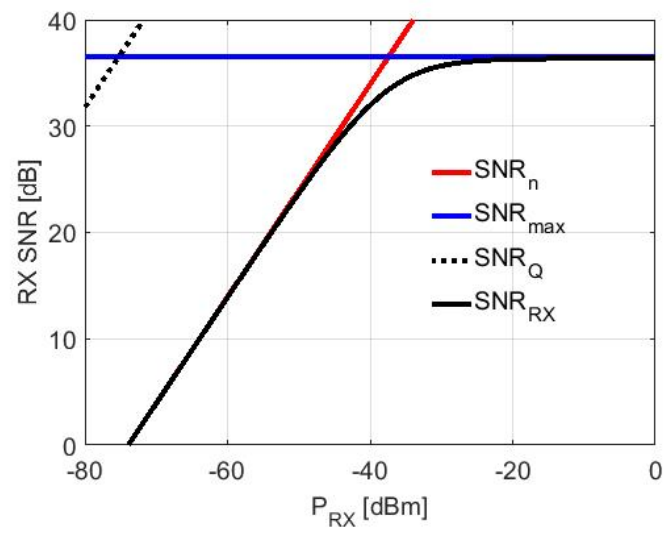

Fig. 3. SNR model of the MT RX.

TABLE V

RECEIVER MODEL PARAMETERS IN COMMUNICATION MODEL

\begin{tabular}{lc}
\hline \hline System bandwidth: $B W$ & $1 \mathrm{GHz}$ \\
NF (DL, UL): $N F_{\mathrm{DL}}, N F_{\mathrm{UL}}$ & $10,8 \mathrm{~dB}$ \\
Maximum RF SNR: $S N R_{\mathrm{Max}}$ & $34 \mathrm{~dB}$ \\
ADC peak-to-peak voltage: $A D C_{V \mathrm{pp}}$ & $2 \mathrm{~V}$ \\
ADC peak-to-peak voltage RMS in $\mathrm{dB}: A D C_{V \mathrm{pp}, \mathrm{dB}}$ & $10 \mathrm{~dB}$ \\
ADC backoff: $A D C_{\mathrm{BO}}$ & $-10 \mathrm{~dB}$ \\
ADC bits & $10 \mathrm{bits}$ \\
ADC resolution: $A D C_{\mathrm{Res}}$ & $62 \mathrm{~dB}$ \\
Receiver maximum gain: $A_{\mathrm{V}, \mathrm{Max}}$ & $60 \mathrm{~dB}$ \\
\hline
\end{tabular}

\section{Distributing SNR between TX and RX for the link}

EVM characterization typically used when characterizing a PA also considers phase responses. However, it is practically too complex to find exact phase relations of all elements in the system. Fortunately, phase responses are very rarely correlated in a large system with large number of non-ideal terms. Therefore a RMS error model is accurate enough for this purpose.

The total SNR of each link, composed of respective SNR/EVM analysis of TX and RX individually can be given as

$$
S N R_{\text {Link }}=\frac{1}{\frac{1}{S N R_{T X}\left(P_{T X}\right)}+\frac{1}{S N R_{R X}\left(P_{R X}\right)}}
$$

where $S N R_{T X}\left(P_{T X}\right)$ is the SNR at the TX output as a function of TX power and $S N R_{R X}\left(P_{R X}\right)$ is the SNR at the RX ADC output as a function of RX power at the antenna connector. Received power, which is dependent on TX power can be simply calculated as

$$
P_{R X}(\mathrm{~dB})=P_{T X}(\mathrm{~dB})-L_{\text {path }}(\mathrm{dB})
$$

where $L_{\text {path }}$ is the loss of the radio channel including array and antenna gains) and $P_{R X}$ and $P_{T X}$ are the RX and TX powers, respectively. Internal partition of RF transceiver is an art of it's own but is based on Friis' formula for noise and combination of other key nonidealities described in detail for example in [17]. To distribute the SNR requirements between the TX and RX, we adopt the LTE BS specifications to create the initial TX EVM requirements (from BPSK to 256QAM). Since the LTE EVM specifications supports only the coded 256QAM (with the EVM of $3.5 \%$ ), we were required to tighten the EVM specification of the highest modulation (the uncoded 256QAM) to the EVM of $2.4 \%$ in order to deliver the highest data rate though the link. Fixed EVM requirements for each modulation 
TABLE VI

TRANSMITTER EVM REQUIREMENTS FOR UNCODED MODULATION SCHEMES

\begin{tabular}{cccccc}
\hline \hline & \multicolumn{5}{c}{ Modulation } \\
\cline { 2 - 6 } & BPSK & QPSK & 16QAM & 64QAM & 256QAM \\
\hline EVM TX [\%] & 17.5 & 17.5 & 12.5 & 5.0 & 2.4 \\
\hline
\end{tabular}

scheme (independent of coding rate) are given in Table VI. The benefit of coding is exploited in the RX sensitivity \& SNR requirements as a function of power as shown in Fig. 4.

Using the TX and RX models, we can describe the TX maximum and RX minimum power levels as a function of data rate, depicted in Fig. 5. The distance between the TX and RX power level pairs dictate the maximum link loss. Although all the modulation points can be utilized in DL, the RF implementation prevents the 256QAM in UL. The maximum link loss for the highest rate in DL is $51.0 \mathrm{~dB}$ (see Fig. 5) without any array gain. As an example, assuming 32 TX and 8 $\mathrm{RX}$ antenna elements may provide the array gain of $>30 \mathrm{dBi}$ for the link, resulting in the maximum range of $11 \mathrm{~m}$ in line-ofsight (LOS) conditions at $27 \mathrm{GHz}$. In that case, the BS and MT beam pattern maxima need to point to each other. Otherwise, the range is smaller. From that perspective, the array control is the key to tolerate the high propagation losses and hence those models are considered in the following Sections. If the range is shorter than the link margin for the maximum rate, the PA power control can be used to reduce output power, and thus both lower power consumption and limit co-channel interference. The RF modelling approaches are illustrated in [16] to highlight the impacts of neglecting RF modelling when estimating range vs. data rate.

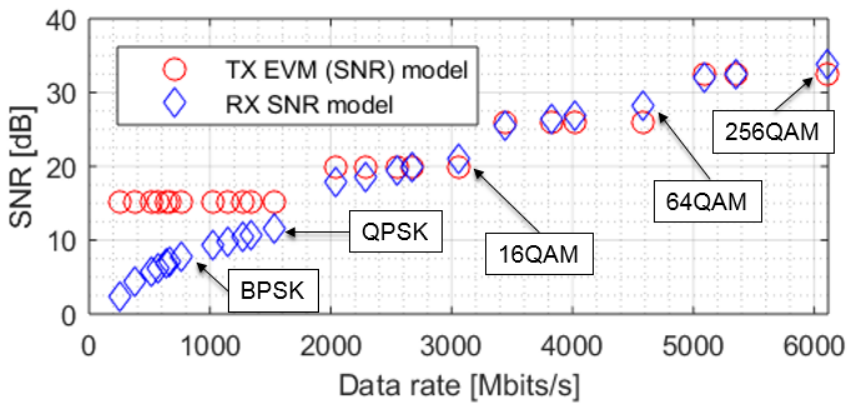

Fig. 4. The total link SNR requirement is distributed between TX and RX SNR models. Modulation points for RX without coding are indicated in the graph.

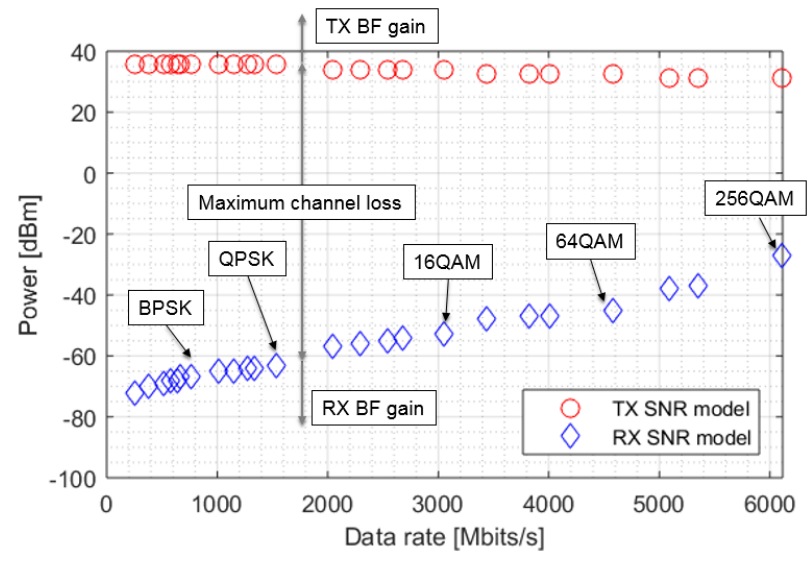

Fig. 5. The maximum TX and minimum RX power levels are connected with the achievable data rates.

\section{ANTENNAS AND PROPAGATION}

\section{A. Antenna Array Models}

Array pattern is a function of an element structure, excitation, phases and geometry. A method of creating array pattern for the system analysis is demonstrated in Fig. 6. First, a singleelement antenna is simulated, in Fig. 6 (a), followed by the calculation of array factor (AF) [18] in Fig. 6 (b). In Fig. 6 (c), the multiplication of the element pattern with the $\mathrm{AF}$ is depicted. Fig. 6 (d) shows the most interesting cutting planes (from the array pattern data that was demonstrated in Fig. 6 (c).

The element pattern in Fig. 7 (a) was taken from the simulations of the widely accepted aperture-coupled structure with a patch radiator. The dual-polarized antenna was designed in CST software, where the pattern information was exported into the Matlab environment. The antenna in Fig. 7 was selected as an example, because the feeding structure and the patch that are separated from each other enables almost independent optimization.

Unlike in BSs with the flexibility to design even hundreds of antenna elements, MTs have notably tighter restrictions for the beam shape. The terminal antennas are typically designed as far as possible from the device body, on a top/bottom end or on the long sides. The implementation complexity emerges from the fact that the array with the feeding network will require relatively large size, but the sides of MT are potentially full of other antennas, connectors and microphones etc., not to mention detrimental hand effect. Although it has been common to claim that higher frequencies enable packing of a large number of antennas, it is expected that any practical array implemented in any commercial MT would not include tens of elements in the near future. Furthermore, MT beams would not be widely directional but may even be a wide beam with just few switched modes for the sake of practical implementation. As an example, we allocate 32 antennas (in the form of $8 \times 4$ ) for BS, while 8 antennas (in the form of $8 \times 1$ ) are employed in each MT.

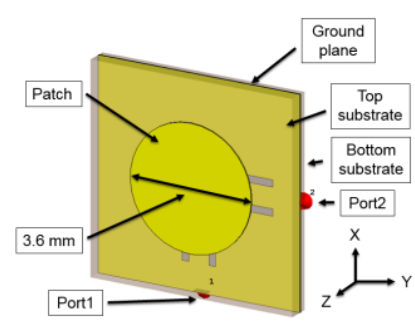

(a)

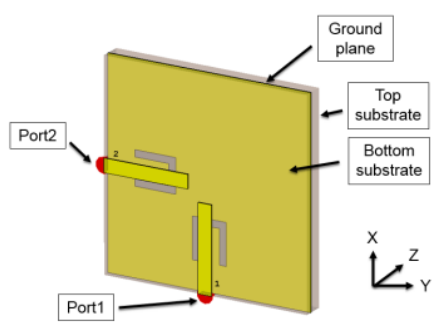

(b)
Fig. 7. A single-element aperture-coupled patch antenna at $27 \mathrm{GHz}$. (a) top view, and (b) back view. The thickness ( $z$-dir) of configuration is $0.62 \mathrm{~mm}$.

\section{B. Propagation Scenario and Map-Based Channel Model}

Link budget is always a function of the target scenario, i.e. propagation environment. The propagation model for $5 \mathrm{G}$ system evaluations is needed to be applicable for the wide range of environments and network topologies. Because of the scalability with the frequency and the applicability for environments, we selected to use map-based model [23], originally created in METIS project. The model employs simplified ray tracing (RT) for resolving the most significant 


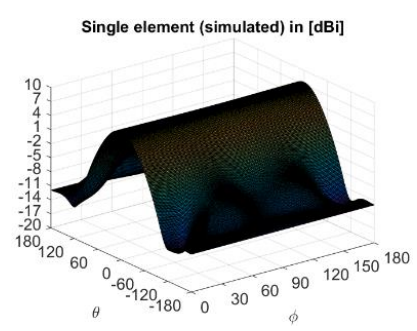

(a)

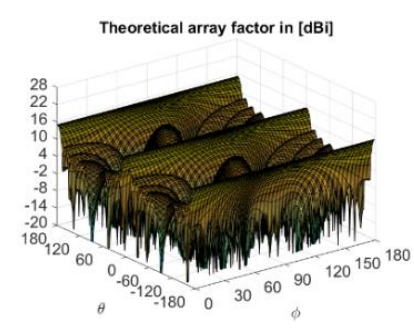

(b)

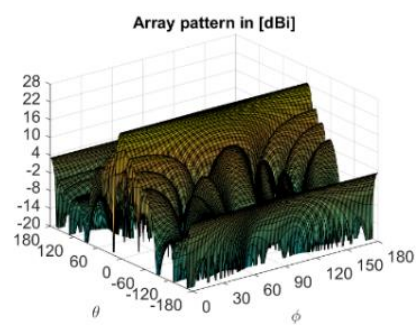

(c)

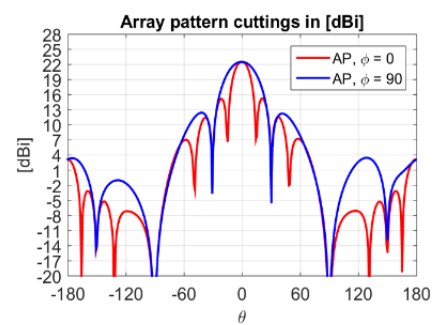

(d)

Fig. 6. Beam pattern estimation for system modelling: a single element pattern for a patch antenna at $27 \mathrm{GHz}$ from CST simulations is imported to the Matlab system simulation environment in (a), multiplied with $4 \times 8$ array factor (AF) shown in (b), in order to provide array radiation pattern in (c) with an ease scalability since the AF handles the geometry of an array. In (d), two most interesting cutting planes are visible from the array pattern data represented in (c).

propagation paths from TX to RX by utilizing the theory of the dominating propagation phenomena, i.e., direct LOS, reflection, diffraction and scattering. The model does not aim to be site specific and complex RT tool, but more like a compromise between the geometry-based stochastic models and RT. The model has been verified already in different frequency bands including $\mathrm{mmW}$ frequencies [24], [25] and it has shown decent modelling accuracy compared to the measurements.

As a reference propagation scenario, indoor office with the rectangular layout of $30 \mathrm{~m} \mathrm{x} 16 \mathrm{~m}$ was selected. The scenario represents possible environment for high data rates targeted for 5G. Typical user traffic is estimated based on the data in [28] (indoor ultra-high broadband access), given in Table VII. The deployment scenario is shown in Fig. 8, where the BS (blue) is located in the corner of office at the height of $2.0 \mathrm{~m}$. The height of MTs vary randomly in the range of 0.5-1.0 m. Simplified three-dimensional description of the environment is built by modelling $1.2 \mathrm{~m}$ high walls with rectangular shapes. Diffraction is modelled by geometric theory of diffraction (GTD), reflection is modelled as single-bounce specular reflection, and direct propagation path via free space propagation with object transmission coefficient. The electrical properties of the materials correspond a plasterboard.

The user positions (red) are visualized in Fig. 8. Simulated propagation paths over the azimuth and elevation planes are shown in Fig. 9. The zero direction is chosen to be towards negative $\mathrm{x}$-axis. The spread of the paths in the azimuth domain indicate that multi-stream transmission required for extreme data rates is possible for specific users if array has enough angular resolution, i.e. enough antenna elements.

TABLE VII

TYPICAL USER TRAFFIC FOR INDOOR DEPLOYMENT SCENARIO

\begin{tabular}{lc}
\hline \hline Connection density $\left[1 / 100 \mathrm{~m}^{2}\right]$ & 7.5 \\
Activity factor $[\%]$ & 30 \\
Area $\left[\mathrm{m}^{2}\right]$ & 480 \\
Dimensions $[\mathrm{m} \cdot \mathrm{m}]$ & $30 \cdot 16$ \\
Number of connections in area & 36 \\
Active connection in area at a time & 12 \\
\hline
\end{tabular}

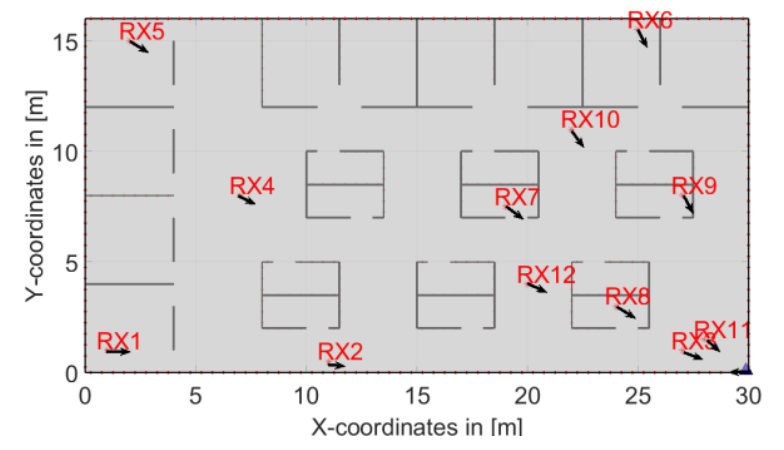

Fig. 8. Indoor open-plan office. Users are arranged in the office area of $30 \cdot 16$ $\mathrm{m}^{2}$ and. Coordinates are defined such that zero direction is in the east and angles run clockwise. The location of BS (TX) is indicated by a blue triangle.

\section{MIMO Channel Generation, Beamforming and Spatial Multiplexing}

In channel modelling, separation of the propagation channel and antennas enables to test the same physical propagation paths with various array geometries and elements. The actual radio channel between each antenna pair can then be calculated based on the given array geometry, polarization, radiation properties and the propagation channel [26]. Radio channel $H_{n_{r}, n_{t}}\left(\tau_{m}, t\right)$ between $n_{r}$ th RX antenna and $n_{t}$ th TX antenna is calculated using (8). In the equation, $F_{\theta, R X, n_{r}}, F_{\phi, R X, n_{r}}, F_{\theta, T X, n_{t}}$ and $F_{\phi, T X, n_{t}}$ denotes the radiation patterns of the corresponding RX and TX antenna elements in vertical and horizontal polarizations, and $\left[\theta_{m}^{A}, \phi_{m}^{A}, \theta_{m}^{D}, \phi_{m}^{D}\right]$ denotes the azimuth and elevation angles of arrival and departure of the $m$ th propagation path, respectively. Three dimensional (3D) position vectors of TX and RX antenna elements are denoted as $d_{T X, n_{t}}$ and $d_{R X, n_{r}}$, and $\hat{r}_{R X}$ and $\hat{r}_{T X}$ are the orthogonal basis vectors of the path angles. $\left[a_{m}^{V V}, a_{m}^{V H}, a_{m}^{H V}, a_{m}^{H H}\right]$ denotes co-polarization and crosspolarization components of the channel. $\lambda_{0}$ and $v$ are the wavelength at the center frequency and $v$ is the Doppler frequency component. Because of static environment, the Doppler term is neglected in our analysis. The geometry is chosen such that BS is pointing towards negative $\mathrm{x}$-axis. For simplicity, all MTs are rotated towards the BS and the effects of random MT orientation is not taken into account. The output is a matrix $\mathbf{H} \in \mathbb{C}^{\mathrm{N}_{\mathrm{r}} \times \mathrm{N}_{\mathrm{t}} \times \mathrm{N}_{\mathrm{D}} \times \mathrm{M}}$, where $M$ denotes the number of time instants, $N_{D}$ denotes the number of delays, $N_{t}$ denotes the 


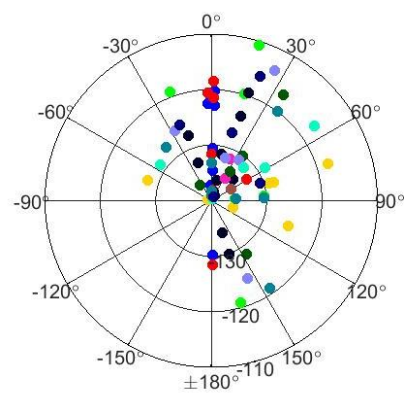

(a)

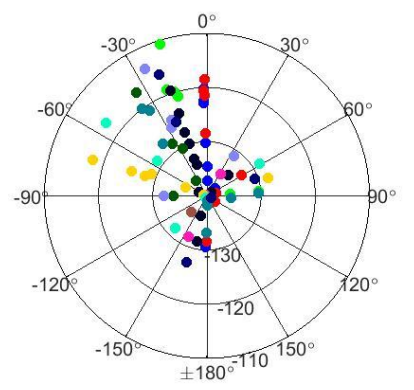

(c)

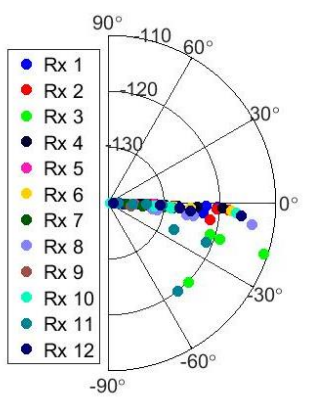

(b)

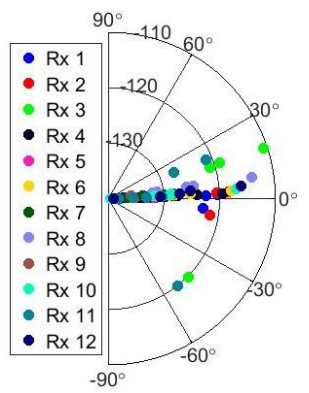

(d)
Fig. 9. Directions of the simulated multipaths within $50 \mathrm{~dB}$ dynamic range from the maxima for (a) TX azimuth, (b) TX elevation, (c) RX azimuth and (c) RX elevation

number of TX and $N_{r}$ number of RX antennas, respectively. The frequency domain channel over the OFDM subcarriers can be then generated by discrete Fourier transform (DFT) over the delay domain. For spatial MIMO processing, the frequency domain is averaged out since per-subcarrier processing is considered to be impractical. For the channel matrix $\mathbf{H}_{\mathrm{AVG}, \mathrm{M}} \mathbb{C}^{\mathrm{N}_{\mathrm{r}} \times \mathrm{N}_{\mathrm{t}}}$, we apply the singular value decomposition (SVD) to produce the eigenmodes of the MIMO channel. For allocating several streams per users, each detectable eigenvalue $\lambda_{i}$ is assumed to be handled as independent link (due to the orthogonality). One should note that because of the nonlinear SNR behavior of TX and RX, and discrete modulation and coding scheme, theoretical power allocation methods such as water-filling etc. are not practical with linear assumptions.

An example of the resolved TX and RX MIMO beams in the azimuth domain for users 5 and 12 are shown in Fig. 10. The beams are scaled with the strengths of the eigenvalues to under stand the condition numbers of the matrices, describing the dynamics between the eigenvalues. SVD clusters all the propagation paths for groups where the beams can be practically steered without interference. Hence, the number of physical propagation paths and possible MIMO beams with a given array configuration are not the same, even though eigenbeams always have a physical meaning, i.e. they are al-

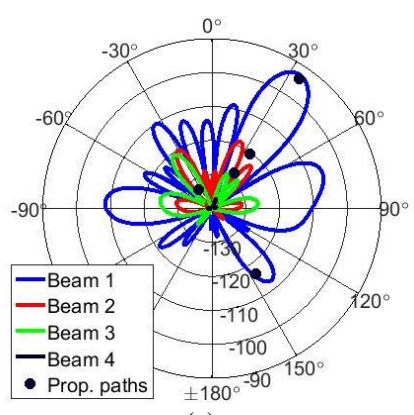

(a)

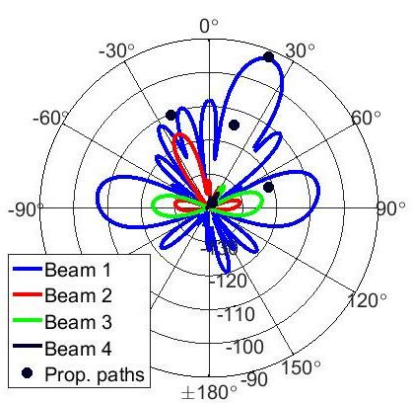

(c)

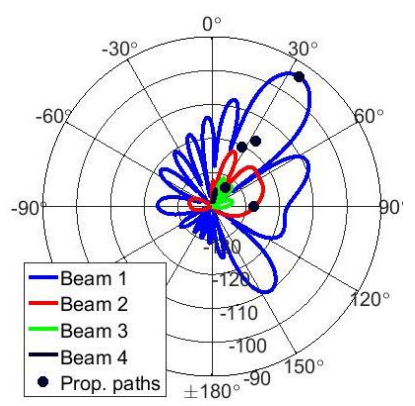

(b)

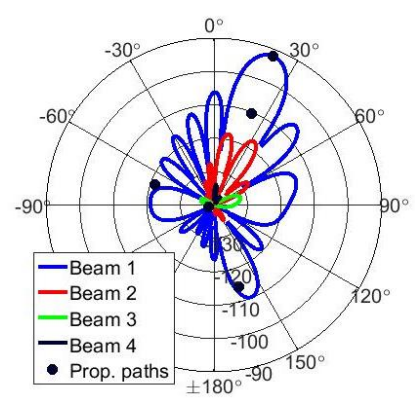

(d)

Fig. 10. (a) and (c) show BS SVD-beams while (b) and (d) corresponding MT SVD-beams for users 5 and 12 , respectively.

ways composed as a linear combination over several physical paths. Eigenbeams are always a combination of TX and RX beamformers which both are needed for providing orthogonal channel. Furthermore, the overall radio channel depends not only on the geometrical properties, but also OFDM parameters, i.e. number of subcarriers and their bandwidth.

\section{Multi-beam RF Link Budget and Performance EVALUATION}

For achieving data rates close to $10 \mathrm{Gbit} / \mathrm{s}$, several SMDS for individual users are needed. In this Section, we combine the transceiver models and link adaptation provided in Section III together with propagation, antenna, and BF models presented in Section IV for creating a link budget for each MIMO-stream.

\section{A. Multi-Stream Link Budget}

In the link budget, it is essential to approach the scenario from two directions: (i) What we can achieve with current transceiver technology in terms of power and linearity? and (ii) What we can achieve with certain link conditions, i.e. in a certain radio channel. These two approaches must be combined when calculating the actual link budget in terms of powers and SNRs. In MIMO, the SNR conditions can be calculated for each beam independently, if we assume the beams to be orthogonal. Hence, each detectable eigenvalue $\lambda_{i}$ represents now one independent link, which we can use as $-L_{\text {path }}(\mathrm{dB})$ in (7).

$$
\begin{gathered}
H_{n_{r}, n_{t}}\left(\tau_{m}, t\right)=\left[\begin{array}{l}
F_{\theta, R X, n_{r}}\left(\theta_{m}^{A}, \phi_{m}^{A}\right) \\
F_{\phi, R X, n_{r}}\left(\theta_{m}^{A}, \phi_{m}^{A}\right)
\end{array}\right]^{T}\left[\begin{array}{cc}
a_{m}^{V V} & a_{m}^{V H} \\
a_{m}^{H V} & a_{m}^{H H}
\end{array}\right]\left[\begin{array}{l}
F_{\theta, T X, n_{t}}\left(\theta_{m}^{D}, \phi_{m}^{D}\right) \\
F_{\phi, T X, n_{t}}\left(\theta_{m}^{D}, \phi_{m}^{D}\right)
\end{array}\right] \\
\times \exp \left(j \frac{2 \pi}{\lambda_{0}} \hat{r}_{R X}\left(\theta_{m}^{A}, \phi_{m}^{A}\right)^{T} d_{R X}, n_{r}\right) \times \exp \left(j \frac{2 \pi}{\lambda_{0}} \hat{r}_{T X}\left(\theta_{m}^{D}, \phi_{m}^{D}\right)^{T} d_{T X}, n_{t}\right) \times \exp \left(j 2 \pi \lambda_{0} v\left(\tau_{m}, t\right)\right)
\end{gathered}
$$


Beam-specific path gains calculated for four best SVDbeams are presented in Fig. 11. Free space path gain (FSPG), together with maximum array gains are presented in the same figure with black dots. Since the propagation scenario is mostly line-of-sight (LOS), the best beams follows approximately the FSPG. Some variation is observed due to the multipath gain/loss and obstruction of the plasterboard walls for some users. Even for a LOS cluster, several paths can be summed constructively or destructively, depending on their phase/delay. In the simulated channel, the first two eigenvalues can be within 10 to $20 \mathrm{~dB}$ for some users, whereas the fourth eigenvalue can be even $55 \mathrm{~dB}$, indicating rank-deficient channel as LOS channels typically are. However, $>15 \mathrm{~dB}$ can still be tolerable condition number, if BF strategy is carefully designed.

The following example is to calculate how much effective isotropic radiated power (EIRP) is required to get $N_{s}$ SMDSs trough, keeping in mind the SNR conditions defined in Section III. From Fig. 5, we can see the maximum allocated data rate for given link loss and the beam specific path gains are presented in Fig. 11. By comparing the maximum channel loss (Fig. 5) and strengths of the eigenvalues (Fig. 11), the maximum available data rate, i.e. largest available modulation scheme is selected. For chosen modulation scheme, the TX power is reduced until the minimum link strength is satisfied. When adding more data streams, naturally more power is also needed. The required EIRPs for achieving rank-N transmission is presented in Fig. 12. Maximum EIRP is calculated based on the $\mathrm{AF}$ equation and element pattern, multiplied with the required TX power. The maximum possible EIRP that can be produced by 32-element patch array, having PAs which are able to produce $34.5 \mathrm{dBm}$ of modulated TX power for each chain, is about $72 \mathrm{dBm}$. However, such EIRP would be only achieved with direct transmission and perfect progressive phase over the antenna elements. Moreover, SVD beamformers have also nonunitary amplitude behavior, because their nature is to orthogonalize the channel. This limits also the actual EIRP of the second, third and fourth eigenbeam, since the full directivity potential of the array cannot be used for such beam shapes.

\section{B. MIMO System Performance}

The user achieved data rates up to rank-4 transmission are presented in Fig. 13. As discussed in the Section II B, maximum data rate that can be achieved with single-stream transmission is 6.11 Gbps. Only users 5 and 9 are not able to use uncoded 256 QAM with the derived SNR specifications. Hence, with properly designed multiantenna transceiver, achieved data rate over single-stream transmission is not limited by the link loss, but RF nonidealities. All the users benefits also from the seconds stream. For users located close to BS, even 256 QAM can be allocated for second stream. However, more variety in rank-2 rata rates over users is seen compared to rank-1 scenario. When increasing the data rate towards rank-3 and rank-4 scenarios, the deviation over the user-experienced data rates is increased. Compared to rank-1 data transmission, multi-stream transmission is more power limited, because it depends both spatial characteristics of the channel and total path loss, i.e. link distance. All of the users are achieving more than $10 \mathrm{Gbps}$ with two or more independent data streams. Furthermore, the nearest users are achieving even more than $20 \mathrm{Gbps}$. This example shows that with proper RF design and large antenna arrays, the

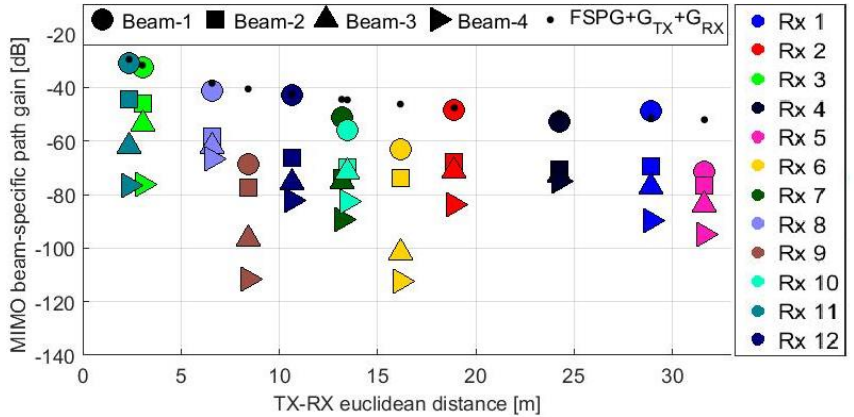

Fig. 11. Eigenvalues of the MIMO channel including the antenna array gain mapped as a function of link distance. Individual users are marked with different colors

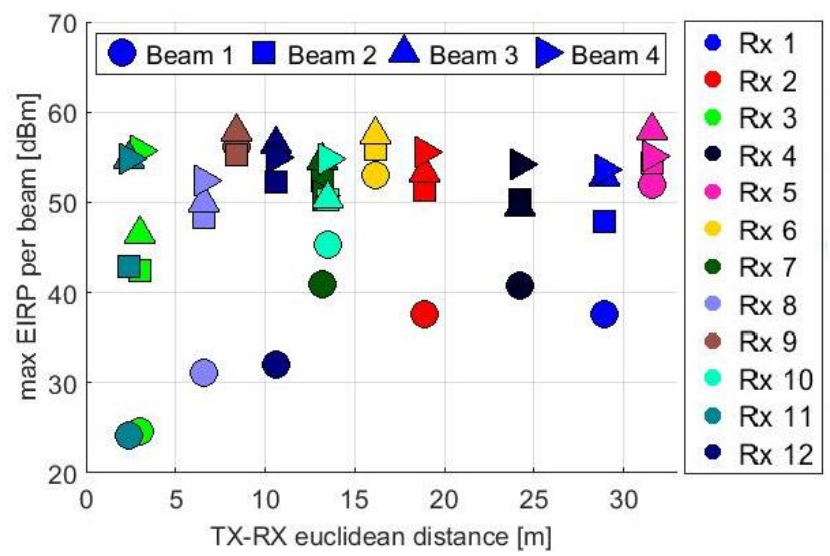

Fig. 12. Required maximum EIRPs of the TX antenna array mapped as a function of link distance for independent MIMO beams. Individual users are marked with different colors.

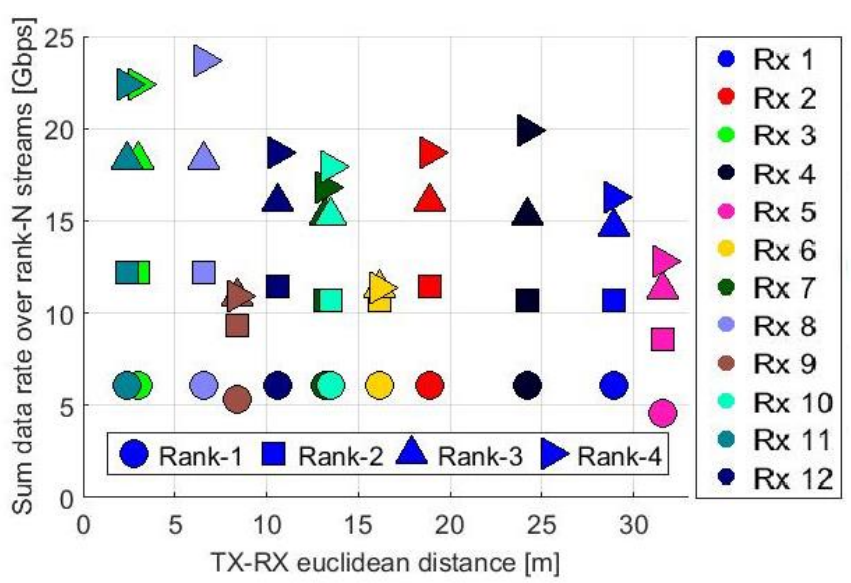

Fig. 13. Sum data rates over N MIMO beams mapped as a function of link distance for independent MIMO beams. Individual users are marked with different colors.

extreme data rates envisioned in $5 \mathrm{G}$ are viable in the example propagation environment.

\section{DISCUSSION AND FUTURE WORK}

In general, the more array gain we can generate, the smaller is the amount of RF power to be produced per link. The maximum required power has also direct relation to the PA size that should be matched reasonably with the antenna element. By separating the propagation channel and antennas, different antenna-geometries can be easily tested to find out the optimal 
power range and spatial resolution with respect to the number of antennas and physical dimensions. Simulation results indicate that MIMO beam angles of arrival from the RX side has huge spread in a realistic environment. A terminal is one of the system level bottlenecks in the case that antenna configuration is not capable of being steered in multiple directions all over the sphere. One should note that the linear array in the terminal edge has the practical scanning limitations to some +/- 30 degrees but the angle variety of the incoming MIMO beams in practical scenario is greatly larger. We expect that mobile array configurations including several small arrays could be highly desirable instead of a single linear array. For a real product, top/bottom ends and long sides are then fully utilized only for $5 \mathrm{G}$ antenna implementations. The optimum way to place antennas with propagation environment for $5 \mathrm{G}$ systems can be figured out with the methodology described in this paper. The sparse-scattering environment raises the need to utilize dual-polarization for MIMO beams to double the capacity. As in the results of Fig. 11, the path gain difference between the strongest and the second beam is large. It is reasonable to assume that in the LOS conditions one could deliver multiple streams also trough two orthogonal polarizations. But the degree to which this could be implemented, needs still research work and measurements.

If one considers a BS that is equipped with tens or even hundreds of antennas, the fully digital architecture is not necessarily the optimal approach due to strong parallelism in data converters and DSP with extensive power consumption. Although the fully digital provides a full degree-of-freedom for using the radio channel, it leads to high system complexity and cost. Full parallelism with the high carrier frequency and wide bandwidth inevitably increases power consumption. Alternative BF strategies such as the hybrid analog/digital that has been recognized attractive by researchers recently [7], [29][31], can also be verified with the methodology presented in this paper. Including multi-user aspects, we need to understand that combination of digital and analog BF is needed for an efficient MIMO system. The digital processing is required for capacity in MU-MIMO and the analog BF is needed for increasing the range. Future studies will include the comparison of hybrid architectures with a fully digital for realistic antenna, propagation and RF implementation models. Also a more comprehensive analysis on the interference vs. capacity tradeoffs in hardware limited RF transceivers could be modelled using the proposed approach.

\section{CONCLUDING REMARKS}

For achieving data rates close to $10 \mathrm{Gbit} / \mathrm{s}$, several SMDS for individual users are needed. We presented methods for combining analysis of signal processing, antennas, RF impairments and radio propagation for defining link-budget towards $5 \mathrm{G} \mathrm{mmW}$ multiantenna systems. The target is to be able to transmit multiple streams for even individual users at 27 $\mathrm{GHz}$ center frequency and over $1 \mathrm{GHz}$ of modulated signal bandwidth. The analysis was derived by first exploiring the performance limits of the modern tranceivers with state-of-theart RF components. Power dependent SNR models were derived for TX and RX and the models were combined to derive a model for minimum link loss vs achivable data rate. The SNR analysis is then combined with map-based propagation model and antenna array to replicate the data rate and power analysis over multiple data streams. The simulations indicated that multi-stream transmission is possible in realistic propagation enviroments and under nonlinear RF conditions. Moreover, with a proper link budget, $10 \mathrm{Gbit} / \mathrm{s}$ data rate can be achieved with minimum of rank-2 transmission. Furthermore, it is shown with a comprehensive but highly abstracted modelling approach that with the realistic RF performance and implementation models, the significant amount of array gain is needed to achieve decent ranges in $5 \mathrm{G}$ at mmWs.

\section{REFERENCES}

[1] H. Q. Ngo, E. G. Larsson, and T. L. Marzetta, "Energy and spectral efficiency of very large multiuser MIMO systems," IEEE Trans. Commun., vol. 61, no. 4, pp. 1436-1449, Apr. 2013.

[2] E. Björnson, E. G. Larsson, and T. L. Marzetta, "Massive MIMO: Ten myths and one critical question," IEEE Commun. Mag., vol. 54, no. 2, pp. 114-123, Feb. 2016

[3] "Cisco, Visual Networking Index: Global Mobile Data Traffic Forecast Update, 2015-2020 White Paper." Available: http://www.cisco.com/c/en/us/solutions/collateral/serviceprovider/visual-networking-index-vni/mobile-white-paper-c11520862.html

[4] "Nokia, 5G Masterplan - Five Keys to Create the New Communications Era." Available: http://networks.nokia.com/innovation $/ 5 \mathrm{~g}$

[5] "Huawei, 5G: A Technology Vision." Available: http://www.huawei.com/5gwhitepaper/

[6] "World Radiocommunication Conference 2015." Available: http://www.itu.int/en/ITU-R/conferences/wrc/2015/

[7] W. Roh, J.-Y. Seol, J. Park, B. Lee, J. Lee, Y. Kim, J. Cho, K. Cheun, and F. Aryanfar, "Millimeter-wave beamforming as an enabling technology for 5G cellular communications: Theoretical feasibility and prototype results," IEEE Commun. Mag., vol. 52, no. 2, pp. 106-113, Feb. 2014.

[8] T. Rappaport, S. Sun, R. Mayzus, H. Zhao, Y. Azar, K. Wang, G. N. Wong, J. K. Schulz, M. Samimi, and F. Gutierrez, "Millimeter wave mobile communications for $5 \mathrm{G}$ cellular: It will work!" IEEE Access, vol. 1, pp. 335-349, May 2013.

[9] O. El Ayach, S. Rajagopal, S. Abu-Surra, Z. Pi, and R. W. Heath Jr., "Spatially sparse precoding in millimeter wave MIMO systems," IEEE Trans. Wireless Commun., vol. 13, no. 3, pp. 1499-1513, Mar. 2014.

[10] Q. Wu, H. Wang, C. Yu, and W. Hong, "Low-profile circularly polarized cavity-backed antennas using SIW techniques," IEEE Trans. Antennas Propag., vol. 64, no. 7, pp. 2832-2839, Jul. 2016.

[11] Z.-C. Hao, X. Liu, X. Huo, and K.-K. Fan, "Planar high-gain circularly polarized element antenna for array applications," IEEE Trans. Antennas Propag., vol. 63, no. 5, pp. 1937-1948, May 2015.

[12] W. Han, F. Yang, J. Ouyang, and P. Yang, "Low-cost wideband and highgain slotted caivty antenna using high-order modes for millimeter-wave application," IEEE Trans. Antennas Propag., vol. 63, no. 11, pp. 46244631, Nov. 2015.

[13] D. Parker, and D. C. Zimmermann, "Phased arrays-Part I: Theory and architectures," IEEE Trans. Microwave Theory Tech., vol. 50, no. 3, pp678-687.

[14] B. Sadhu, et al., "A $28 \mathrm{GHz} 32$-element phased-array transceiver IC with concurrent dual polarized beams and 1.4 degree beam-steering resolution for 5G communication," Digest of the IEEE International Solid-State Circuits Conference (ISSCC), San Francisco, USA, pp. 128-120, 5-9 February 2017.

[15] "3GPP TR 38.913 Study on Scenarios and Requirements for Next Generation Access Technologies." Available: http://www.3gpp.org/DynaReport/38913.htm

[16] T. Tuovinen, N. Tervo, and A. Pärssinen, "RF system requirement analysis and simulation methods towards $5 \mathrm{G}$ radios using massive 
MIMO," in Proc. $46^{\text {th }}$ Europ. Microw. Conf. (EuMC), London, United Kingdom, pp. 142-145 Oct. 4-7, 2016.

[17] B. Razavi, RF microelectronics, 2nd Edition, Pearson Education, Inc., 2012.

[18] C. Balanis, Antenna Theory: Analysis and Design. Wiley, 2012.

[19] N. Klemmer, et al., “A 45nm CMOS RF-to-bits LTE/WCDMA FDD/TDD 2×2 MIMO base-station transceiver SoC with 200MHz RF bandwidth," Digest of the IEEE International Solid-State Circuits Conference (ISSCC), San Francisco, USA, pp. 164-165, 1-4 February 2016.

[20] T. Georgantas, et al., "A $13 \mathrm{~mm} 2 \quad 40 \mathrm{~nm}$ multiband GSM/EDGE/HSPA+/TDSCDMA/LTE transceiver," Digest of the IEEE International Solid-State Circuits Conference (ISSCC), San Francisco, USA, pp. 160-161, 22-26 February 2015.

[21] Qorvo, "27.5 - $31 \mathrm{GHz} 8 \mathrm{~W}$ GaN Power Amplifier”, TGA2595-CP datasheet, July, 2016.

[22] S. Shakib, H.-C. Park, J. Dunworth, V. Aparin, and K. Entesari, "A 28 $\mathrm{GHz}$ efficient linear power amplifier for $5 \mathrm{G}$ phased arrays in $28 \mathrm{~nm}$ bulk CMOS," in Proc. IEEE Int. Solid-State Circuits Conf. (ISSCC), San Francisco, USA, pp. 219-223,, Jan. 31-Feb. 4, 2016.

[23] ICT-317669 METIS project, "METIS channel models, Deliverable D1.4 V3," Jun. 2015. [Online]. Available: Available: https://www.metis2020.com/wpcontent/uploads/deliverables/METIS_D1.4_v1.0.pdf

[24] I. Carton, Wei Fan, P. Kyösti and G. F. Pedersen, "Validation of 5G METIS map-based channel model at mmwave bands in indoor scenarios, "10th European Conference on Antennas and Propagation (EuCAP), Davos, 2016, pp. 1-5.

[25] A. Roivainen, P. Kyösti, V. Hovinen, C. F. Dias, N. Tervo, M. Sonkki, G. Fraidenraich and M. Latva-aho, "Validation of Deterministic Radio Channel Model by $10 \mathrm{GHz}$ Microcell Measurements, "22th European Wireless Conference, Oulu, Finland, 2016, pp. 1-6.

[26] A. Roivainen, C. Ferreira Dias, N. Tervo, V. Hovinen, M. Sonkki, and M. Latva-aho, "Geometry-based stochastic channel model for two-storey lobby environment at $10 \mathrm{GHz}$," IEEE Trans. Antennas Propag., vol. 64, no. 9, pp. 3990-4003, Jun. 2016.

[27] T. Tuovinen, N. Tervo, H. Pennanen and A. Pärssinen, "Providing 10 Gbit/s in downlink to a mobile terminal with practical array design beamforming aspects by using orthogonal MIMO beams," in Proc. European Wireless (EuWI), Oulu, Finland, pp. 229-234, May 18-20, 2016.

[28] NGMN, "NGMN 5G white paper," Feb. 2015. [Online]. Available: https://www.ngmn.org/uploads/media/NGMN_5G_White_Paper_V1_0. pdf

[29] S. Han, C.-L. I, Z. Xu, and C. Rowell, "Large-scale antenna systems with hybrid analog and digital beamforming for millimeter wave 5G," IEEE Commun. Mag., vol. 53, no. 1, pp. 186-194, Jan. 2015.

[30] F. Sohrabi, and W. Yu, "Hybrid digital and analog beamforming design for large-scale MIMO systems," in Proc. IEEE Int. Conf. Acoustics, Speech and Signal Process. (ICASSP), Australia, South Brisbane, pp. 2929-2933, Apr. 19-24, 2015.

[31] A. Alkhateeb, O. El Ayach, G. Leus, and R. W. Heath Jr., "Channel estimation and hybrid precoding for millimetre wave cellular systems," IEEE J. Sel. Topics Signal Process., vol. 8, no. 5, pp. 831-846, Oct. 2014.

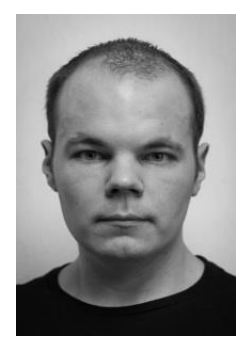

Tommi Tuovinen received the B.Sc, M.Sc and D.Sc degrees in Technology at Centre for Wireless Communications (CWC) from the University of Oulu, Finland in 2010, 2011 and 2014, respectively. From the beginning of 2015 until the end of 2016, he worked as a Post-Doctoral Researcher at CWC. Currently, he is working at Convergentia Ltd as antenna specialist.

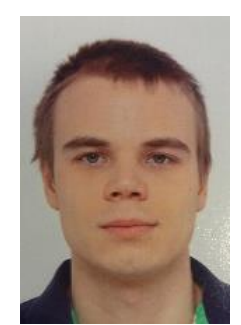

Nuutti Tervo (S'16) received the B.Sc. (Tech.) and M.Sc. (Tech.) degrees in electrical engineering from the University of Oulu, Finland, in 2014. He joined the Centre for Wireless Communications (CWC), University of Oulu, in 2014.

His previous works included radio channel measurements and modelling for submillimeter-wave frequencies. Currently, he is pursuing the Ph.D. degree on RF power amplifiers and their nonlinear impact on 5G millimeter-wave MIMO systems.

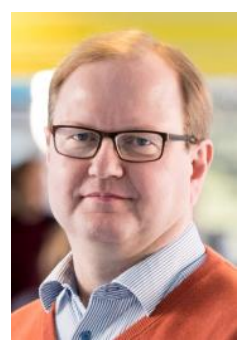

Aarno Pärssinen (S'95-M'01-SM'11) received the M.Sc., Licentiate in Technology and Doctor of Science degrees in electrical engineering from the Helsinki University of Technology, Finland, in 1995, 1997, and 2000, respectively.

From 1994 to 2000 he was with Electronic Circuit Design Laboratory, Helsinki University of Technology, Finland, working on direct conversion receivers and subsampling mixers for wireless communications. In 1996, he was a Research Visitor at the University of California at Santa Barbara. From 2000 to 2011 he was with Nokia Research Center, Helsinki, Finland. During 2009-2011 he served as a member of Nokia CEO Technology Council. From 2011 to 2013, he was at Renesas Mobile Corporation, Helsinki, Finland working as a Distinguished Researcher and RF Research Manager. From October 2013 to September 2014 he was an Associate Technical Director at Broadcom, Helsinki, Finland. Since September 2014 he has been with University of Oulu, Centre for Wireless Communications, Oulu, Finland where he is currently a Professor. His research interests include wireless systems and transceiver architectures for wireless communications with special emphasis on the RF and analog integrated circuit and system design.

Aarno Pärssinen has authored and co-authored one book, one chapter of a book, more than 50 international journal and conference papers and holds several patents. He served as a member of the technical program committee of Int. Solid-State Circuits Conference in 2007-2017, where he was the chair of European regional committee in 2012-13, and the chair of the wireless sub-committee in 2014-2017. Since July 2015 he is serving as Solid-State Circuits Society representative for IEEE for IEEE $5 \mathrm{G}$ initiative. 